\title{
Suicídio de internos em um hospital de custódia e tratamento*
}

\author{
Suicide among inmates hospitalized in a Forensic Psychiatric Hospital
}

Elizabete Rodrigues Coelho', Fernanda Azevedo', Gabriel José Chittó Gauer ${ }^{2}$, Alfredo Cataldo Neto ${ }^{3}$

\section{RESUMO}

Objetivo: Descrever o perfil dos internos suicidas do Instituto Psiquiátrico Forense (IPF) de Porto Alegre em duas décadas. Método: Foi realizado um estudo retrospectivo dos registros dos internos com óbito por suicídio. Os dados foram analisados por meio da estatística descritiva. Resultados: Dos 20 casos de suicídio, 70\% cumpriam medida de segurança, 70\% eram réus primários, $80 \%$ dos crimes era contra a pessoa; a maioria (45\%) dos suicídios foi cometida durante a madrugada e $40 \%$ dos casos ocorreram com internos que estavam de 1 a 9 anos na instituição; 90\% eram do sexo masculino, 55\% tinham idade entre 20 e 39 anos, 70\% eram solteiros, 60\% não tinham filhos, 85\% eram naturais do interior do RS, 25\% não tinham profissão, 84,2\% tinham até o Ensino Fundamental; 55\% dos casos tinham diagnóstico de esquizofrenia. Em 75\% dos casos a morte ocorreu por enforcamen-

\section{Palavras-chave}

Suicídio, Instituto

Psiquiátrico Forense, direito penal, psiquiatria forense.

\section{Keywords}

Suicide, Forensic Psychiatric Institute, criminal law, forensic psychiatry. to. Conclusões: Os dados apontam para o seguinte perfil: homem, solteiro, sem filhos, do interior do Estado, com baixa qualificação profissional e pouca escolaridade. Os fatores psicossociais encontrados foram transtorno mental grave, uso de drogas e/ou álcool, baixo suporte social e familiar e tentativas anteriores de suicídio.

\section{ABSTRACT}

Objective: To describe the profile of suicides inmates at a Forensic Psychiatric Institute in Porto Alegre from two decades. Method: It was a retrospective study of the inmates records with a history of death by suicide. The data were analyzed through descriptive statistics. Results: There was a total of 20 cases of suicide, $70 \%$ were involuntary commitment, $70 \%$ were primary defendants, $80 \%$ of crimes against persons, the majority (45\%) of suicides were committed in the daybreak period and $40 \%$ of the cases occurred in inmates that were from 1 to 9 years in the institution. They also had the following characteristics: 90\% were male, 55\% were between 20 and 39 years old, $70 \%$ single, $60 \%$ childless man, $85 \%$ of the subjects were originally from small towns, 25\% had no profession, $84.2 \%$ had primary schooling level. The diagnostic in 55\% of the cases was schizophrenia. In 75\% of cases the death occurred by hanging. Conclusion: The data point to the profile of a single and childless man, originally from small towns, with low professional qualification and schooling. Psychosocial factors were severe mental disorder and drug or alcohol use, low social and family support, previous suicide attempts, high impulsivity and aggression.

1 Pontifícia Universidade Católica do Rio Grande do Sul (PUC-RS), Faculdade de Psicologia.

2 Pontifícia Universidade Católica do Rio Grande do Sul (PUC-RS), Faculdade de Psicologia e Faculdade de Direito, Programa de Mestrado e Doutorado em Ciências Criminais.

Recebido em 


\section{INTRODUÇÃO}

O suicídio é uma autoagressão intencional extrema que, além de ser uma expressão inequívoca de sofrimento individual intenso, gera impacto profundo nos familiares, nos internos e na equipe de atendimento de saúde e da custódia. Segundo Cassorla', a intencionalidade refere-se à intensidade do desejo da pessoa em terminar com a própria vida e as consequências do ato indicariam a letalidade. Angerami-Camon ${ }^{2}$ afirma que o suicida não busca o desaparecimento real; a opção pelo suicídio é uma tentativa de livrar-se de conflitos e sofrimentos.

Nas populações de ambientes prisionais, o suicídio é uma causa frequente de morte. Segundo a Organização Mundial da Saúde (OMS), 20006,8, elas enfrentam dificuldades de toda ordem e se encontram em situações de vulnerabilidade por causa de vários fatores ambientais, além dos sentimentos de desamparo, desesperança, agressividade, impulsividade, culpa e da presença de doença mental, que também pode ser desencadeante para o comportamento suicida nesses ambientes. Os hospitais psiquiátricos forenses apresentam muitas dificuldades em virtude da falta de programas específicos de prevenção em saúde e prevenção do suicídio, bem como pela falta de profissionais, de material de higiene, de remédios e de programas efetivos de reinserção social dos doentes mentais que cometeram um delito 3

Este trabalho, portanto, teve como objetivo determinar o perfil dos internos que cometeram suicídio no Instituto Psiquiátrico Forense Doutor Maurício Cardoso (IPF), em Porto Alegre, Rio Grande do Sul, no período de 1985 a 2004.

\section{MÉTODOS}

Este estudo foi autorizado pelo Dr. Rogério G. Cardoso, diretor-geral do Instituto Psiquiátrico Forense Dr. Maurício Cardoso, apreciado pelo Comitê de Ética em Pesquisa da Pontifícia Universidade Católica do Rio Grande do Sul e autorizado sob o Ofício no 686/05-CEP, em 4 de agosto de 2005.

Foi realizado um estudo retrospectivo com o fim de investigar as características demográficas, clínicas e criminológicas dos internos que cometeram suicídio no Instituto Psiquiátrico Forense Dr. Maurício Cardoso, no período de 1985 a 2004. Buscaram-se nos registros dos internos os casos em que o óbito foi descrito como "morte por suicídio". Desses casos, foram coletados dados dos registros clínicos e administrativos com as seguintes variáveis: gênero, idade, situação conjugal, naturalidade, profissão, filhos, grau de instrução, situação jurídica, delito, diagnóstico, sintomatologia imediatamente anterior ao suicídio, método empregado no suicídio, data, hora e local do suicídio, história de tentativas anteriores, história familiar de suicídio, relações familiares, bem como cartas ou bilhetes para comunicar a decisão de morrer.

No período de janeiro de 1985 a dezembro de 2004 foram registradas 197 mortes no instituto. A população em estudo compreende 20 internos. Do total de mortes, sete prontuários em que havia suspeita de suicídio foram excluídos do estudo por falta de dados específicos sobre as circunstâncias em que as mortes ocorreram. Os sujeitos desta pesquisa constavam como internos na instituição por determinação judicial e em decorrência de qualquer um dos motivos previstos em lei.

Os dados coletados foram analisados por meio de estatística descritiva, com a utilização do programa estatístico Pacote Estatístico para Ciências Sociais (Statistical Package for the Social Sciences - SPSS). De maneira geral, foram construídas distribuições de frequência simples, para todas as variáveis apuradas.

Não foram feitos testes estatísticos de hipóteses, por estarem sendo estudados todos os casos de suicídio ocorridos no IPF, no período de interesse, constituindo a populaçãoalvo do estudo, e não apenas uma amostra.

\section{RESULTADOS}

Do total de 197 mortes ocorridas no IPF entre janeiro de 1985 e dezembro de 2004, houve o registro de 20 casos de suicídio consumado (que representam 10,1\% do total de mortes). Foram considerados todos os casos em que os sujeitos se encontravam em avaliação e em tratamento e/ou em custódia do Estado na instituição e que morreram por suicídio, ocorrido dentro ou fora da instituição (fuga ou saída autorizada).

Com relação à situação jurídica, no momento da morte $70 \%$ dos sujeitos se encontravam cumprindo medida de segurança, 25\% estavam detidos preventivamente (não tinham uma condenação) e $5 \%$ encontravam-se presos e foram encaminhados para tratamento e avaliação psiquiátrica. Do total de casos, 70\% eram réus primários.

A maioria dos casos (80\%) apresentava o delito de crime contra a pessoa (homicídio, tentativa de homicídio e lesões corporais). Em segundo lugar (20\%) aparecem os crimes sexuais (estupro e atentado violento ao pudor). O delito contra a propriedade (incêndio) e o delito de tráfico foram identificados em 5\% dos casos, cada um. Em alguns casos, ocorreu mais de um delito.

No que tange ao horário do óbito, em 25\% dos casos não foi possível obter tais dados. Nas ocorrências em que esse dado foi registrado, verificou-se que a madrugada foi o horário em que houve o maior número de suicídios (45\%), seguida do turno da tarde (20\%) e do período da manhã (10\%). 
Do total dos casos de suicídio analisados, 35\% ocorreram no primeiro ano de internação; 25\% deles foram registrados entre o primeiro e trigésimo dia, a partir da internação. Oito casos (40\%) ocorreram no período de um a nove anos de internação.

Dos 20 casos estudados, $90 \%$ dos sujeitos eram do sexo masculino, a maioria (55\%) tinha idade entre 20 e 39 anos e $30 \%$ estavam com idade entre 20 e 29 anos.

Com relação ao estado civil, $70 \%$ dos internos que cometeram suicídio eram solteiros, 20\% eram casados e 10\% foram classificados como separados no momento de ingresso na instituição. Do total de suicidas, 60\% não tinham filhos e $85 \%$ dos sujeitos eram naturais do interior do Estado do Rio Grande do Sul.

Relativamente à ocupação, os dados apontaram para um baixo nível de qualificação profissional. Em 25\% dos casos, os indivíduos não tinham uma profissão definida; $20 \%$ tinham uma ocupação ligada ao meio rural; $10 \%$ eram jornaleiros e de serviços gerais; 10\% tinham profissões ligadas ao lar; 15\% eram aposentados (a aposentadoria em todos os casos foi efetivada pela condição de doente mental).

Dos 20 casos, 36,8\% dos sujeitos eram analfabetos e 47,4\% tinham como grau de instrução o Ensino Fundamental. Cabe salientar que nesses casos estão incluídos sujeitos que sabiam apenas escrever o nome e que não eram considerados analfabetos para fins de registro na instituição. O Ensino Médio compreende 15,8\% dos casos. Não havia nenhum sujeito com educação superior.

Os sujeitos deste estudo receberam, ao todo, 28 diagnósticos; alguns receberam mais de um diagnóstico. $\mathrm{O}$ diagnóstico mais frequente foi de esquizofrenia, encontrada em 55\% dos casos, com o subtipo paranoide presente em 40\% dos casos. O diagnóstico de transtorno de personalidade paranoide foi exarado em 15\% dos casos. Os transtornos mentais e de comportamento decorrentes do uso de álcool estavam representados em 10\% do total. Convém ressaltar que a dependência de álcool e/ou drogas, como uma patologia secundária ao diagnóstico principal, foi relatada em $50 \%$ dos casos. Os diagnósticos de retardo mental moderado e de epilepsia estiveram representados, cada um, em $10 \%$ dos casos.

Dos 20 casos analisados, em 45\% não constavam dados sobre a ocorrência ou não de tentativas anteriores de suicídio. Dos casos restantes, em 50\% houve a ocorrência de tentativas anteriores de suicídio e em $5 \%$ os sujeitos não haviam tentado o suicídio. Quanto ao número de tentativas anteriores, metade (50\%) havia tentado uma vez; 30\%, duas vezes; 10\%, três vezes; 10\% tinham mais de três tentativas anteriores ao suicídio.

Quanto ao meio empregado, em $75 \%$ dos casos a morte ocorreu por enforcamento. Em 10\%, a opção adotada foi jogar-se de altura. Os casos de afogamento, automu- tilação e ingestão de medicamentos representam 5\% do total de suicídios.

Nos documentos consultados não foram encontrados, em nenhum dos casos, registros acerca da presença ou não de comportamento suicida familiar. No entanto, no que tange ao suporte social e familiar, os documentos consultados apresentam registros de afastamento da família e sentimentos de rejeição, tristeza, desesperança e falta de perspectivas futuras. Em apenas dois casos houve um bilhete explicando os motivos do suicídio aos familiares.

\section{DISCUSSÃO}

Este estudo procurou identificar as variáveis e os fatores envolvidos no suicídio de internos no Instituto Psiquiátrico Forense Maurício Cardoso. Até o momento, não foram encontrados estudos brasileiros específicos sobre o suicídio dessa população.

As pesquisas sobre suicídio em ambientes de reclusão apresentam características peculiares e algumas limitações. Lloyd $^{4}$ refere que, em alguns dos estudos realizados, foram incluídos, em uma mesma pesquisa, casos de suicídio e de tentativas de suicídio. Os estudos foram baseados em populações de réus primários, sem uma sentença condenatória; e outros, ainda, foram feitos com presos já condenados. Além disso, essas pesquisas se referem a diferentes culturas, o que leva a dificuldades de comparações. Assim, os dados encontrados no presente estudo foram discutidos, em parte, com base nos estudos realizados em ambientes de custódia internacionais e, também, no trabalho de Menezes ${ }^{5}$, referente às características dos pacientes em tratamento no IPF.

Ocorreram 20 casos de suicídio entre 1985 e 2004. Desses, 70\% eram relacionados a pacientes internados em medida de segurança, $25 \%$ a réus sem uma sentença transitada em julgado e $5 \%$ a presos já condenados. Do total de casos, 70\% eram de réus primários.

Taxas elevadas de mortes por suicídio no ambiente penitenciário são referidas pela OMS. Nos estudos realizados em diversos ambientes penitenciários, essas taxas oscilaram entre três e dez vezes mais que as da população geral, dos países pesquisados ${ }^{6}$. A taxa média de suicídios, em relação ao total de mortes na instituição, no período de 1985 a 2004, foi de 10,1\%. Essa taxa difere, por exemplo, daquela encontrada nas prisões australianas, que foi de $46,6 \%$ de suicídios, em relação ao total de mortes no ambiente penitenciário?. Com relação a esse dado, deve-se considerar que muitos fatores podem influenciar nas taxas de suicídio de maneiras diferentes, dependendo do sistema correcional, da população de internos, das circunstâncias locais e culturais e do acesso à saúde básica e à saúde menta| ${ }^{8}$. 
Os reclusos do referido estudo cometeram suicídio predominantemente por enforcamento (75\%), fato que demonstra a forte intencionalidade e a alta agressividade do ato?.

Os horários de maior incidência foram os da madrugada. 0 horário entre as 24 horas e o amanhecer também foi apontado por Lester ${ }^{10}$ como o de maior incidência de suicídios. A menor vigilância e o número ainda mais reduzido de funcionários concorrem para esse achado.

Green et al. ${ }^{9}$, ao pesquisarem as variáveis criminológicas e os suicídios cometidos por réus primários nas prisões canadenses, verificaram, em relação aos delitos praticados, que em primeiro lugar estavam os crimes contra a pessoa e, em seguida, os crimes sexuais. Folino et al. ${ }^{11}$ referem em seu estudo que, em relação à maioria dos sujeitos que se encontravam cumprindo pena em uma penitenciária, a maior incidência foi de crimes contra a propriedade. Nos casos pesquisados, a maioria (80\%) cometeu crimes contra a pessoa (homicídio, tentativa de homicídio, lesões corporais), e em 55\% desses casos, o crime cometido foi o homicídio. Podese inferir, concordando com Johnson et al.12, que a violência do ato criminoso e a violência que configura o ato suicida, na população internada, são fruto da alta impulsividade e da agressividade dirigidas aos outros e a ela mesma.

A vulnerabilidade consequente do ingresso em uma instituição de custódia desempenha importante papel no comportamento suicida, principalmente por causa da situação de insegurança do recluso acerca da sua situação jurídica. Segundo o estudo de Folino et al."1, 60\% dos reclusos cometeram suicídio nos três primeiros meses de ingresso. No IPF, os suicídios ocorreram, em 35\% dos casos, no primeiro ano de internação e, em $25 \%$ dos casos, entre o primeiro e o trigésimo dia. Menezes ${ }^{5}$ encontrou, entre a população do IPF, um tempo médio de internação de 7,12 anos, com um desvio-padrão de 6,99 anos.

As pesquisas sobre fatores de risco, para comportamento suicida, têm apontado uma correlação elevada entre o suicídio e a presença de algum transtorno psiquiátrico. Na população reclusa em geral e no caso de pacientes psiquiátricos forenses o risco tende a ser maior ${ }^{6}$. Em seu estudo, Marcus Alcabes ${ }^{13}$ referem uma alta incidência de doenças mentais na população encarcerada. Encontraram no sistema penitenciário norte-americano 52\% dos suicidas com transtorno psiquiátrico e 46\% com uma história anterior de tratamento psiquiátrico.

Stalenheim ${ }^{14}$ encontrou principalmente o diagnóstico de transtorno de personalidade borderline nos casos de suicídio no ambiente psiquiátrico forense. $O$ estudo dos casos aponta para a existência de patologias graves, bem como a presença do uso abusivo de álcool e/ou drogas. A maioria dos internos recebeu o diagnóstico de esquizofrenia (55\%); $40 \%$ desses casos são do subtipo paranoide. Apesar de o diagnóstico de transtornos mentais pelo uso de álcool ter sido verificado em $10 \%$ dos casos, a dependência do álcool e/ou drogas foi relatada, como decorrente da patologia principal, em 50\% dos casos. Esse dado vem reforçar a importância da intoxicação por álcool ou drogas, como um fator que pode aumentar ou precipitar o risco de comportamento suicida.

Entre os estudos realizados nos diversos ambientes penitenciários revisados neste trabalho, poucos foram realizados, especificamente, em um hospital psiquiátrico forense. Nas penitenciárias do Texas, os suicidas receberam, em primeiro lugar, os diagnósticos relativos aos transtornos de humor (64\%), seguidos dos transtornos de personalidade (56\%) e dos transtornos psicóticos $(44 \%)^{15}$. No ambiente psiquiátrico forense, Apter et al. ${ }^{16}$ dizem que os pacientes não apresentam uma prevalência de transtornos afetivos nem uma correlação significativa entre tristeza e risco de comportamento suicida. Essas patologias são encontradas entre os pacientes psiquiátricos em geral que cometeram o suicídio.

O levantamento acerca da ocorrência de tentativas anteriores de suicídio indica uma alta incidência (55\%), chegando a ocorrer, em 10\% dos casos, mais de três tentativas. Meleiro e Teng $^{17}$ dizem que os sobreviventes de uma tentativa de suicídio apresentam um risco elevado de repetir esse comportamento e completar o suicídio.

Quanto ao gênero, os suicídios, na atual pesquisa, foram praticados por indivíduos do sexo masculino, em sua maioria (90\%). Os estudos norte-americanos e australianos apontam a população masculina, nos ambientes de custódia, como mais vulnerável ao suicídio. Os estudos epidemiológicos em geral também indicam o alto índice de suicídios entre os homens. No Brasil, a ocorrência de suicídio é de duas a três vezes mais frequente com relação a homens do que a mulheres ${ }^{13,17}$.

No tocante ao estado civil e à presença ou não de fiIhos, os internos suicidas, em sua maioria, foram classificados como solteiros e sem filhos. O elevado percentual de indivíduos classificados como solteiros, quando do ingresso na instituição, pode não corresponder à verdadeira situação conjugal deles, pois, mesmo tendo um companheiro(a), podem ter sido classificados como solteiros(as). A presença de filhos é apontada como um fator de proteção em relação ao comportamento suicida. O senso de responsabilidade despertado com a presença de crianças pode ser "uma razão de viver" 17 .

A maioria dos suicidas nos ambientes penitenciários possui histórias de muitas privações. São jovens sem uma profissão definida, com baixa escolaridade, que têm muitas dificuldades de relacionamento e incapacidade para encontrar soluções para seus problemas ${ }^{18}$. O presente estudo corrobora com esse autor, pois a maioria dos internos possuía um nível baixo de escolaridade e qualificação profissional.

Os dados psicossociais levantados neste trabalho sugerem que a maioria daqueles que cometeram suicídio 
encontrava-se afastada de seus familiares ou mantinha relações conflitantes com eles. Muitos apresentavam comportamento agressivo, sentimentos de tristeza, de rejeição, de desesperança e de culpa, principalmente em relação à família. O afastamento da família pode ocorrer pelo distanciamento físico, pois o IPF é a única instituição de custódia e tratamento do Estado. Neste estudo, $85 \%$ dos sujeitos que cometeram suicídio eram originários do interior do Estado.

Apesar das limitações desta pesquisa, entre elas a falta de um grupo adequado com o qual fosse possível comparar os dados encontrados, os resultados demonstram que estar preso é um fator de risco importante para comportamento suicida, mas estar custodiado e ter uma patologia mental eleva ainda mais esse risco. Os resultados indicam, ainda, a necessidade da inserção de medidas preventivas que levem em conta as características específicas dessa população.

\section{CONCLUSÃO}

A investigação possibilitou identificar as principais características demográficas, clínicas e criminológicas dos internos que cometeram suicídio no Instituto Psiquiátrico Forense Dr. Maurício Cardoso entre 1985 e 2004. As características comuns encontradas apontaram para o perfil de um homem, solteiro, sem filhos, do interior do Estado, com baixa qualificação profissional e baixa escolaridade. Os fatores psicossociais de risco na maioria dos casos foram: a presença de um transtorno mental grave e o uso abusivo de drogas e/ou álcool, baixo suporte social e familiar e tentativas anteriores de suicídio. A maioria desses sujeitos foi acusada de ter praticado crimes contra a vida, principalmente o homicídio, e morreram por enforcamento durante a madrugada.

Conhecer tais características é importante para melhorar a capacidade de detectar e de lidar com o interno com risco de suicídio, planejar e estabelecer métodos e programas de prevenção e de intervenção, além da necessidade de acompanhamento dos profissionais que lidam com essa população. Entende-se que um programa de treinamento para os profissionais envolvidos nos cuidados dessa clientela pode melhorar a detecção e o manejo do risco de suicídio dos internos. Por fim, sugere-se que novas pesquisas científicas possam ser direcionadas à identificação daqueles internos que tentaram o suicídio na instituição, e que não se suicidaram, com a finalidade de melhor detectar, instalar e reforçar os fatores protetores.
Instituto Psiquiátrico Forense Doutor Maurício Cardoso, Porto Alegre, Rio Grande do Sul.

* Dissertação de mestrado defendida no Programa de Ciências Criminais da Faculdade de Direito da Pontifícia Universidade Católica do Rio Grande do Sul (PUC-RS).

\section{REFERÊNCIAS}

1. Cassorla RMS. Suicídio e autodestruição humana. In: Werlang BG, Botega NJ, organizadores. Comportamento suicida. Porto Alegre: Artmed; 2004. p. 21-33.

2. Angerami-Camon VA. Suicídio: fragmentos de psicoterapia existencial. São Paulo: Pioneira; 1997.

3. Souza CAC. Doentes mentais em prisões e em casas de custódia e tratamento: um grande e permanente desafio. [acessado em nov. 2005]. Disponível em: http://www.polmed.com.br.

4. Lloyd C. Suicide and self-injury in prison: a literature review. Home Office Research and Planning Unit Report. London: Her Majesty's Stationery Office; 1990. [acessado em jun. 2005]. Disponível em: http://www.csc-scc.ca/.

5. Menezes R. Homicídio e esquizofrenia: estudo de fatores associados [dissertação]. Porto Alegre: Universidade Federal do Rio Grande do Sul (UFRGS); 2001.

6 WHO. Preventing suicide: a resource for prison officers. Mental and behavioural disorders. Department of mental health. World Health Organization. Geneva; 2000.

7. Dalton V. Australian Institute of Criminology. Suicide in prison 1980 to 1998: National Overview, no 126 - trends \& issues in crime and criminal justice. August, 1999.

8. WHO. Banco de dados. Relatórios sobre suicídios, 2000 e 2001. [acessado em jan. 2009]. Disponível em: http://www.who.int.word.

9. Green C, Kendall K, Andre G, Looman T, Polvi N. A study of 133 suicides among Canadian federal prisoners: prison violence and self-injury. Med Sci Law. 1992;4(3). [acessado em jun. 2005]. Disponível em: http://www.csc-scc.ca/crd/forum/e043/3043i.htm.

10. Lester D. Suicide behind bars: prediction and prevention. Pennsylvania: The Charles Press, Publishers, Inc.; 1993. p. 1-14.

11. Folino J0, Marchiano SE, Wilde AS. Suicidios en convictos bonaerenses. Rev Argent Psiquiatria. 2003;XIV:286-91.

12. Johnson C, Smith J, Crowe C, Donovan M. Suicide among forensic psychiatric patients. Med Sci Law. 1993;33(2):137-43.

13. Marcus P, Alcabes P. Characteristics of suicides by inmates in an urban jail. Hosp Community Psychiatry. 1993;44(3):256-61.

14. Stalenheim EG. Relationships between attempted suicide, temperamental vulnerability and violent criminality in a Swedish forensic psychiatric population. Eur Psychiatry. 2001;16(7):386-94.

15. He XY, Felthous AR, Holzer CE, Nathan P, Veasey S. Factors in prison suicide: one year study in Texas [resumo]. J Forensic Sci. 2001. [acessado em 20 out. 2003]. Disponível em: http:// www.scielo.medline.

16. Apter A, Kotlerm M, Sevy S, Plutchik R, Brown SL, Foster H, et al. Correlates of risk of suicide in violent and nonviolent psychiatric patients. Am J Psychiatry. 1994;51(9):1316-23.

17. Meleiro A, Teng CT. Fatores de risco de suicídio. In: Meleiro A, Teng CT, Wang YP. Suicídio: estudos fundamentais. São Paulo: Segmento Farma; 2004. p. 109-131.

18. Liebling A. Suicides in young prisoners: a summary. Death Stud. 1993;17(5):381-9. 